
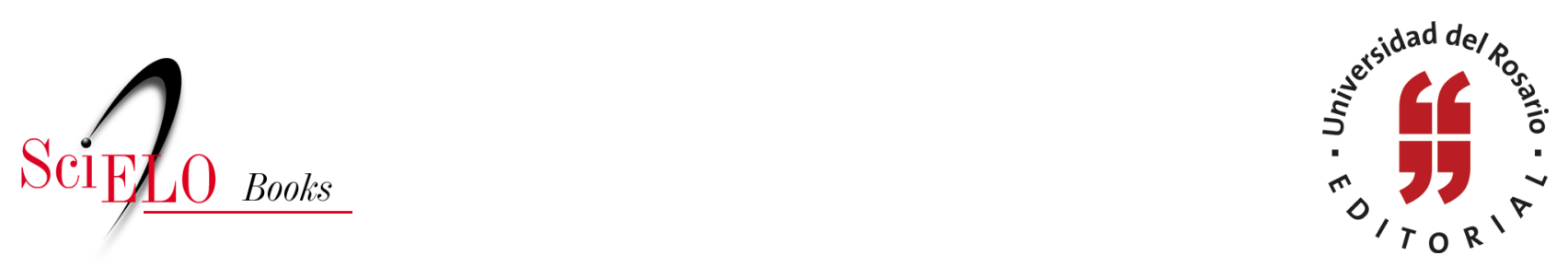

\title{
1. Aproximación a los estudios sobre clientelismo en Colombia análisis de la matriz metodológica
}

\author{
Laura Daniela Guerrero García
}

\section{SciELO Books / SciELO Livros / SciELO Libros}

GUERRERO GARCÍA, L.D. Aproximación a los estudios sobre clientelismo en Colombia: análisis de la matriz metodológica. In: Clientelismo político, ¿desviación de la política o forma de representación?: Estado del arte sobre las aproximaciones al clientelismo en Colombia 1973-2011 [online]. Bogotá: Editorial Universidad del Rosario, 2014, pp. 15-23. Opera prima collection. ISBN: 978-958-738-552-6. https://doi.org/10.7476/9789587385526.0003.

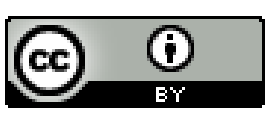

All the contents of this work, except where otherwise noted, is licensed under a Creative Commons Attribution 4.0 International license.

Todo o conteúdo deste trabalho, exceto quando houver ressalva, é publicado sob a licença Creative Commons Atribição 4.0.

Todo el contenido de esta obra, excepto donde se indique lo contrario, está bajo licencia de la licencia $\underline{\text { Creative }}$ Commons Reconocimento 4.0 . 


\section{Aproximación a los estudios sobre clientelismo en Colombia: análisis de la matriz metodológica}

Este capítulo describe las fuentes analizadas sobre el clientelismo político en Colombia, explicando su ubicación temporal e importancia teórica. Identifica, además, la existencia de diferentes enfoques y formas de aproximación al fenómeno.

La década del setenta es el punto de partida, momento en el que se observa una recurrencia en el empleo del término "clientelismo" en remplazo de otras categorías como las de "caudillismo", "caciquismo"y "patrimonialismo". En esta década, los estudios de clientelismo se caracterizaron por tomar fuerza tanto en Europa como en los Estados Unidos de Norte América, EE. UU., dando lugar a debates entre enfoques que, por supuesto, llegaron a Colombia y se manifestaron en las perspectivas y problemáticas abordadas.

En el país, los estudios sobre clientelismo representan un corpus de producción académica sostenida desde $1974 .{ }^{3}$ En este año, el Centro de Investigaciones y Educación Popular, Cinep, emprendió una serie de esfuerzos académicos con el fin de "indagar por las bases socioeconómicas del comportamiento político en el agro

${ }^{3}$ Comparar Fernán E. González, “Clientelismo y administración pública”, Enfoques colombianos, (1980): 68 . 
Colombiano" ${ }^{4}$ a partir del análisis teórico y los estudios de caso regionales. Este trabajo, liderado por Néstor Miranda Ontane$\mathrm{da},{ }^{5}$ se enmarcó en el enfoque marxista, perspectiva desde la cual se pretendía superar las dificultades del enfoque funcionalista. ${ }^{6}$

Lo expresado no niega la existencia de estudios anteriores: en 1973 se puede localizar el antecedente internacional más cercano en la tesis doctoral de Steffen Walter Schmidt en la Universidad de Columbia. ${ }^{7}$ Walter Schmidt revisó el sistema político colombiano y consolidó un marco conceptual sobre el clientelismo, introducido al tema por Orlando Fals Borda y León Bramson. ${ }^{8}$ Además de esta obra y de las publicaciones del Cinep, para la época se contó con otros aportes históricos, teóricos y sociológicos de autores

${ }^{4}$ Ver Fernán E. González, "Aportes al diálogo entre historia y ciencia política. Una contribución desde la experiencia investigativa en el Cinep”(Historia Crítica, 2004), 28.

${ }^{5}$ Comparar Néstor Miranda Ontaneda, Clientelismo y dominio de clase (Bogotá: Cinep, 1977).

${ }^{6}$ Miranda Ontaneda llegó a Colombia luego de publicar en Bamberg Klientelismus und koloniale Abhängigkeit. Eine ethnosoziologische Analyse d. Repartimiento-Encomienda-Systems aufd.Antillen,1492-1525 (Elclientelismo y la dependencia colonial: Un análisis etno-sociológico del sistema de repartimiento-encomienda de las Antillas, 1492-1525) (Bamberg: Heidelberg, 1968). Entre 1975 y 1978 dirigió una investigación en el Cinep que buscaba romper con el enfoque ahistórico y funcionalista de la época. Cuatro publicaciones con estudios de caso, de corte marxista, fueron el resultado. Estos asumían al clientelismo como un mecanismo de instrumentalización del ser humano para la conservación del poder de las élites políticas. Su importancia radicó en la inscripción del país en un tema en furor en Europa y EE. UU. y en la aplicación rigurosa de un marco teórico para la realidad colombiana. Los cuatro estudios fueron el de Alejandro Reyes, Latifundio y poder político (Bogotá: Cinep, 1978); el de Eloísa Vasco Montoya, Clientelismo y minifundio (Bogotá: Cinep, 1978); el de Jorge Ramírez Valenzuela, Producción arrocera y clientelismo (Bogotá: Cinep, 1978); y la compilación de artículos publicados por la Fundación Friedrich Naumann, compilados por Néstor Miranda Ontaneda, Clientelismo (Bogotá: Fundación Friederich Naumann, 1980).

${ }^{7}$ Comparar Steffen Walter Schmidt, "Political Clientelism in Colombia" (tesis de doctorado, University Microfilms International, Londres, 1972).

${ }^{8}$ Sociólogo profesor de la Universidad de Harvard. 
nacionales tales como Fernando Guillén Martínez y de escuelas anglosajonas entre cuyos representantes se puede citar a Malcolm Deas, Frank Safford y Catherine LeGrand. ${ }^{9}$ Así, el clientelismo empezó a ser objeto de estudio en Colombia a partir de los años setenta, de manera paralela a los estudios del clientelismo europeos y estadunidenses. Se trata de una historia conectada y concomitante, cuyo agente difusor fue, sin duda, Miranda Ontaneda.

La producción de los setenta se caracterizó por estar enmarcada dentro de los enfoques funcionalista y marxista. Los principales voceros fueron Steffen Walter Schmidt ${ }^{10}$ y Néstor Miranda Ontaneda. Las variables empleadas respondían a una trasformación del sistema político colombiano. De ahí que estos trabajos abordaron el problema desde: i) la observancia de la burocracia aplicada al régimen del Frente Nacional; ii) el sistema productivo agrario de la época; iii) el desarrollo de la economía capitalista; y iv) el problema del poder político manejado por las élites bipartidistas.

En la década de los ochenta se presentó una explosión de estudios de caso sobre clientelismo. Así, los esfuerzos del Cinep dieron sus frutos en forma de estudios regionales marxistas, dentro de los cuales están los trabajos de Eloísa Vasco, Alejandro Reyes, Jorge Ramírez, Mario Alviar, Fernán González, Jaime Arocha, Jaime Echavarría y Julio Hálaby y Eduardo Díaz. ${ }^{11}$ Su aporte fue

\footnotetext{
${ }^{9}$ Comparar Fernán. E. González, “Aportes al diálogo entre historia y ciencia política. Una contribución desde la experiencia investigativa en el Cinep"(Historia Crítica, 2004), 29.

${ }^{10}$ Comparar Steffen W. Schmidt, "Bureaucrats as Modernizing Brokers? Clientelism in Colombia" (Comparative Politics 6, 1974), 425-450 y Steffen W. Schmidt, "Political Clientelism in Colombia" (1972).

${ }^{11}$ Comparar Jaime Arocha, "Clientelismo, gasteo y violencia”, Enfoques colombianos, (1980); Fernán González, "Clientelismo y administración pública”, Enfoques colombianos, (1980); Mario Alviar, “Clientelismo y Participación política”, Enfoques colombianos, (1980);
} 
significativo para la comprensión del funcionamiento del poder local, en regiones como Sucre, Quindío, Chocó y Boyacá.

Para finales de los años ochenta aparecieron enfoques que antes de preguntarse por las relaciones de explotación del campesinado, causadas por las formas de producción pre-capitalista, se cuestionaban por el problema de la democracia en Colombia. En este contexto y con una mirada más institucionalista, Rodrigo Losada introdujo la problemática del voto clientelista en las elecciones populares, ${ }^{12}$ y John Sudarsky abordó la desigualdad del ingreso desde la formación de cooperativas. ${ }^{13}$ Desde entonces, el sistema político colombiano, la crisis de los partidos políticos y los cambios institucionales producidos, a partir de las reformas de 1968 y 1991, pasaron a ser el centro de atención de los autores.

En los años noventa, la obra de Francisco Leal y Andrés Dávila, ${ }^{14}$ desde un enfoque estructural-funcionalista, marcó un hito en el abordaje del clientelismo al comprenderlo como el articulador de todo el sistema político colombiano y no sólo como un mecanismo que instrumentalizaba individuos en función de votos y favores. Con la vigencia de la Constitución de 1991, se presentó una explosión de estudios institucionalistas y con enfoques anclados a la cultura política, a partir de los cuales se empezarían

Néstor Miranda Ontaneda, "El poder político en Colombia”, Enfoques Colombianos,(1980); Eduardo Díaz Uribe, El clientelismo en Colombia: Un estudio exploratorio (Bogotá: Áncora Editores, 1986); Jaime Echavarría Córdoba, y Julio C. Hálaby Córdoba, Sociología política de comicios y clientelismo (Chocó: Universidad Tecnológica del Chocó, 1988).

${ }^{12}$ Comparar Rodrigo Losada Lora, Clientelismo y elecciones: tres modelos explicativos del comportamiento electoral colombiano (Bogotá: Pontificia Universidad Javeriana, 1984).

${ }^{13}$ Comparar John Sudarsky, Clientelismo y desarrollo social (Bogotá: Tercer Mundo Editores, 1988).

${ }^{14}$ Comparar Francisco Leal Buitrago y Andrés Dávila Ladrón de Guevara, Clientelismo, el sistema politico y su expresión regional (Bogotá: Tercer Mundo Editores, 1991). 
a abordar otros aspectos del clientelismo tales como: la participación, la representación y la formación de ciudadanía. Dichos estudios estarían liderados por autores tales como Francisco Gutiérrez Sanín, Andrés Dávila, Miguel García y Rocío Rubio. ${ }^{15}$

En este recorrido se observa que desde finales de la década de los ochenta hay una apertura a examinar el clientelismo desde diferentes enfoques (funcionalista, marxista, estructural-funcionalista, el socio-antropológico, con anclajes en la cultura política, y el institucionalista). Estos observan al fenómeno como modo de distribución de bienes y servicios, ${ }^{16}$ forma de participación y representación; ${ }^{17} \mathrm{o}$ bien como la peor deformación de la democracia, antesala de la corrupción política ${ }^{18} \mathrm{y}$ producto de la crisis de los partidos tradicionales. ${ }^{19}$

${ }^{15}$ Comparar Francisco Gutiérrez Sanín, “Clientelismo y sus enredos”; Andrés Dávila Ladrón de Guevara, "Clientelismo, intermediación y representación política en Colombia: ¿Qué ha pasado en los noventa?” (Estudios Politicos, 1999); Miguel García Sánchez, ¿Ciudadanía avergonzada? Democracia local y construcción de ciudadanía en Bogotá (Bogotá: Universidad de los Andes y Universidad Nacional de Colombia, 2003); y Rocío Rubio Serrano, No hay paraísos sino los perdidos: Historia de una red clientelista en Bogotá (Bogotá: Universidad Nacional de Colombia, 2003).

${ }^{16}$ Duarte, Educación.

${ }^{17}$ Comparar Fernando Escalante, “Clientelismo y ciudadanía en México: apuntes sobre la conceptualización de las formas de acción política” (Analisis Político, 1995).

${ }^{18}$ Comparar Roll, David Alberto, "Elclientelismo: corrupción a tres bandas", Sindéresis, (2000), 114-128.

${ }^{19}$ Comparar John D. Martz, The Politics of Clientelism: Democracy E the State in Colombia (New Brunswick, New Jersey: Transaction Publishers, 1997). 


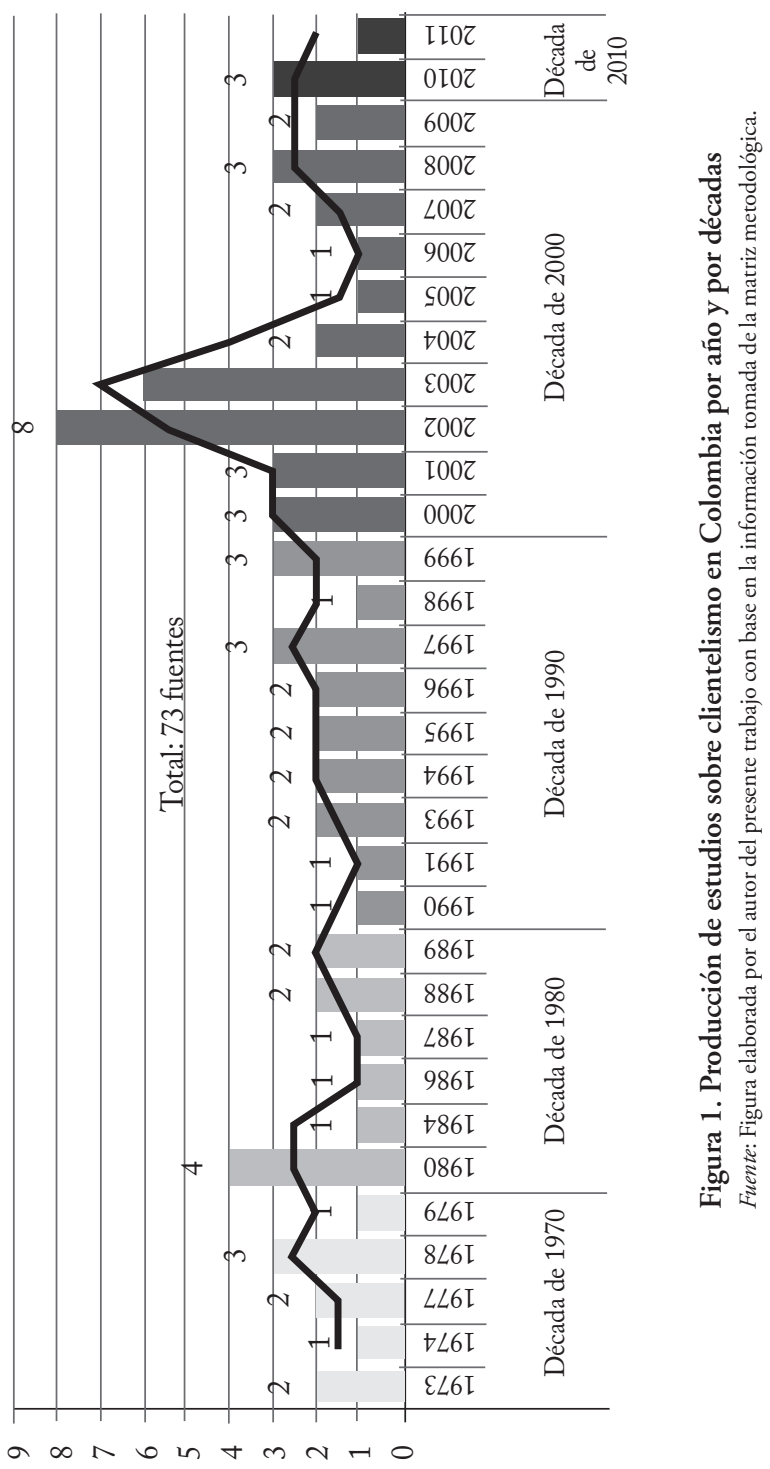


Adicionalmente, la matriz metodológica, elaborada para dar cuenta de este estado del arte (ver anexo 3), indica a manera de conclusión que en Colombia aparecieron los estudios pioneros sobre clientelismo en la década de los setenta. En la siguiente década, se observa un terreno fértil para los estudios regionales de caso. Desde 1993, se identifica que hay una tendencia a producir anualmente al menos un trabajo sobre clientelismo. Entre el 2002 y el 2003, evidencia la máxima producción de las cuatro décadas revisadas (ver figura 1). Por último, señala un declive de los estudios sobre clientelismo a partir de la segunda década del siglo xxI.

Los hallazgos de la matriz permiten establecer que en la década de los setenta los dos enfoques predominantes fueron el marxista y el funcionalista, teniendo en cuenta que el estructuralismo representa una vertiente del marxismo. Una década más tarde, se observó la desaparición del enfoque funcionalista y el auge del enfoque marxista. Simultáneamente, se mantuvo constante la cantidad de estudios de corte institucionalista, a la vez que emergió tímidamente la perspectiva socio-antropológica.En los noventa se observó un declive en el empleo del enfoque marxista; no obstante, algunas de sus ideas siguen prevaleciendo, lo que ha evitado su completa desaparición. El enfoque hegemónico en esta década fue el institucionalista; por otro lado, el estructural-funcionalista mostró sus primeras apariciones, mientras que el enfoque socio-antropológico mostró un ligero incremento. Para comienzos del siglo xxI, el enfoque predominante fue el institucionalista, le siguió el socio-antropológico y el estructural-funcionalista, pese a que se presentaron eventuales trabajos desde el enfoque marxista. 


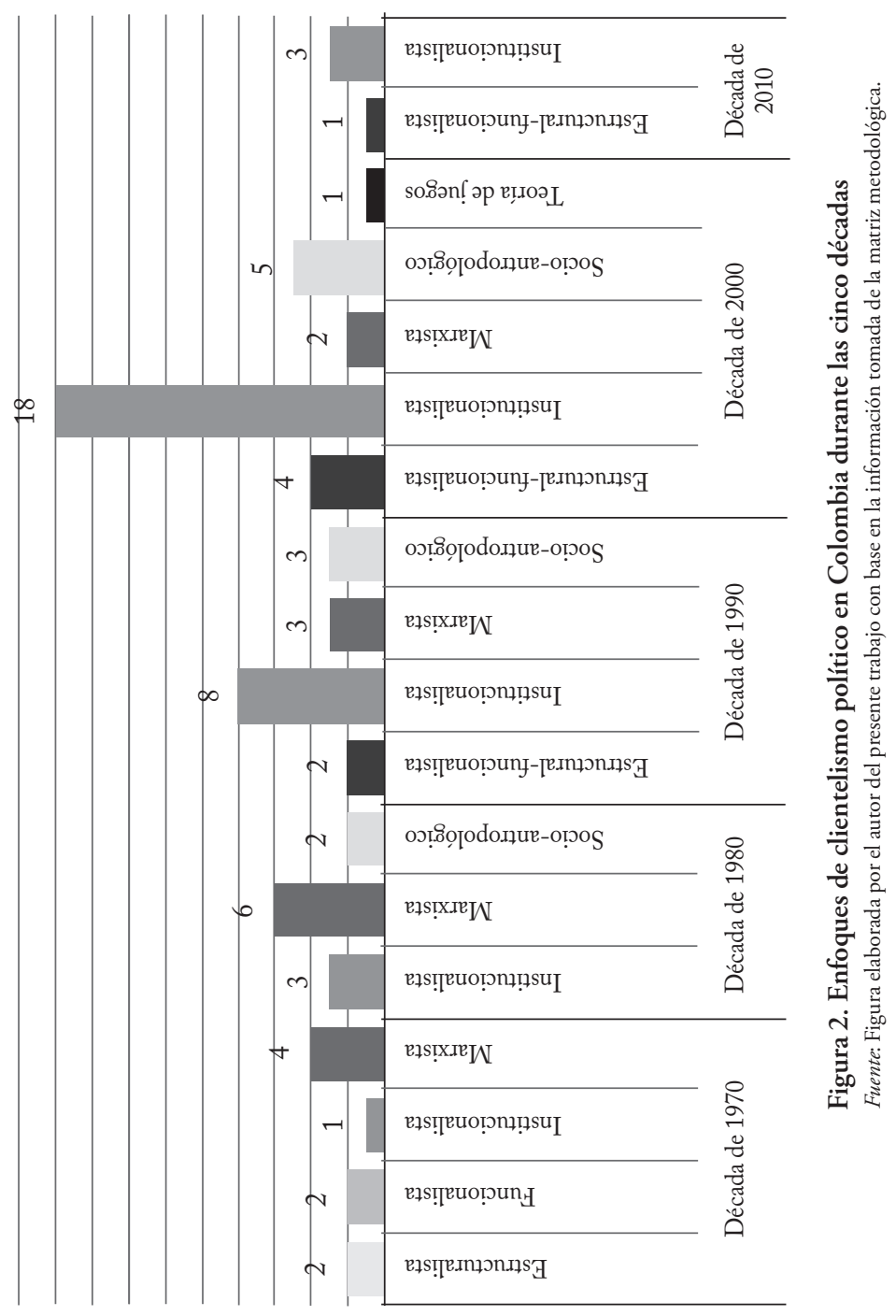


Identificar los límites teóricos de cada trabajo es una tarea que requiere claridad sobre los enfoques, ya que no todos los autores manifiestan explícitamente sus adscripciones. Sin embargo, las categorías enunciadas y los argumentos presentados son la primera clave para evidenciar el marco conceptual soporte de cada estudio. Este ejercicio se realizó, en un primer nivel de análisis, en la construcción del presente estado del arte.

A continuación, además de explicar los criterios de clasificación de las obras, se buscará hacer una exposición de las trasformaciones de cada enfoque teórico, así como de una serie de eventos diagnósticos que alimentaron la discusión a propósito del tema. Vale la pena aclarar que la selección de los eventos diagnósticos que se presentan a continuación es producto de la literatura revisada. Finalmente, se pretende mostrar el cambio que ha sufrido la forma de abordar el fenómeno en Colombia. 